Norois

Environnement, aménagement, société

$195 \mid 2005 / 2$

L'appropriation de l'espace : sur la dimension spatiale des inégalités sociales et des rapports de pouvoir

\title{
Usage et symboliques des représentations spatiales en Roumanie post-communiste
}

Uses and Symbolisms of Spatial Representations in Post-Communist Romania

\section{Emmanuel Bioteau}

\section{OpenEdition}

\section{Journals}

Édition électronique

URL : https://journals.openedition.org/norois/565

DOI : 10.4000/norois.565

ISBN : 978-2-7535-1543-7

ISSN : 1760-8546

\section{Éditeur}

Presses universitaires de Rennes

\section{Édition imprimée}

Date de publication : 1 juin 2005

Pagination : 93-107

ISBN : 978-2-7535-0123-2

ISSN : 0029-182X

Référence électronique

Emmanuel Bioteau, « Usage et symboliques des représentations spatiales en Roumanie postcommuniste », Norois [En ligne], 195 | 2005/2, mis en ligne le 08 août 2008, consulté le 13 janvier 2022. URL : http://journals.openedition.org/norois/565 ; DOI : https://doi.org/10.4000/norois.565

Ce document a été généré automatiquement le 13 janvier 2022.

(c) Tous droits réservés 


\title{
Usage et symboliques des représentations spatiales en Roumanie post-communiste
}

Uses and Symbolisms of Spatial Representations in Post-Communist Romania

\author{
Emmanuel Bioteau
}

\section{NOTE DE L'ÉDITEUR}

Cet article a été reçu le 16 novembre 2004 et définitivement accepté le 31 mai 2005.

1 Par quels moyens la Roumanie pouvait-elle réaliser sa sortie du communisme ? De ce large questionnement ${ }^{1}$ résulte un besoin de regard sur les transformations de l'espace social et de la société en général. Des modalités de regroupements, que les autorités communistes s'étaient attachées à limiter, furent de nouveau rendues possibles. Rapidement, des groupes se forment sur les bases d'héritages historiques et ethnolinguistiques partagés. Le renforcement de certaines Eglises facilite ces recompositions sociales (Vernicos-Papageorgiu et Rey, 1994). Il en va de même de la résurgence de régionalismes, exacerbés par des différences intra-étatiques se renforçant.

2 En cette société et en ces espaces partiellement formatés par l'idéal communiste, il subsiste peu de référents, visuels ou autres, de la présence en certains lieux de certaines populations. Les recompositions socio-spatiales induites par le changement de régime ont pour effet premier de former ce type de repères, de fonder de nouvelles limites, de nouveaux ancrages. Ce marquage de l'espace contribue tant à la réaffirmation qu'à la fondation d'identités spécifiques.

Dans ces conditions de redéfinition de l'espace public, comme du bien privé, l'identité redevient bien ce « produit d'une situation sociale dans laquelle interviennent l'auto- et 
l'hétéro- définition du groupe » (Schnapper, 1998). Le groupe n'existerait-il alors que parce que rendu visible depuis l'extérieur?

\section{Se représenter l'espace, le marquer, dans la période post-communiste}

4 Les représentations territoriales et sociales des citoyens roumains ont été fortement modifiées durant la période communiste. Le projet de constitution d'une société uniforme promettait d'effacer toute singularité socio-spatiale, au bénéfice d'un idéal théoriquement partagé, en réalité imposé. La révolution de 1989 agence un nouvel ordre, a priori commun, car choisi par tous. Ces dispositions sont aptes à fonder une société citoyenne, en l'occurrence unifiée. En somme, l'actuelle mutation de système se doit de fonder une société nouvelle.

5 Le recouvrement des libertés individuelles permet la résurgence de regroupements identitaires. Ces rassemblements attestent de la division de la population roumaine. Ceux-ci répondent d'une histoire partagée par divers citoyens et sont organisés par une ou plusieurs conditions communes de lieu, langue ou confession. Les modalités d'expression des groupes ainsi constitués varient et s'ordonnancent, depuis une base associative $^{2}$ jusqu'à une insertion dans le champ politique. Au final, des réseaux sociaux se constituent, lesquels sont orchestrés par des critères d'appartenance.

6 Hors le lien social, la dimension spatiale des rapports sociaux fait elle aussi l'objet d'une redéfinition, à la mesure des bouleversements politiques. De nombreux représentants de groupes considèrent que leurs actions renouvellent des configurations territoriales antérieures à l'avènement du communisme. Ainsi, du rattachement à la Roumanie de la Transylvanie - à laquelle est associée le Banat - naît le souvenir de singularités historiques et spatiales majeures: certaines formes de multiculturalité, un pluriconfessionnalisme avéré ou encore une avance économique et technologique. En ces territoires identifiés de longue date, la période d'entre-deux-guerres, voire le souvenir de l'Empire austro-hongrois font, pour certains, office de références. Pourtant, de nombreux territoires administratifs ont été remodelés par les autorités communistes. Des migrations intérieures, liées à l'emploi, ont été imposées. Des départs pour l'extérieur de certains ressortissants ont été tolérés, voire encouragés ${ }^{3}$. Toute initiative naissant dans l'immédiat post-communisme ne peut désormais s'effectuer qu'à l'échelle des petites villes (et communes) ou des quartiers. Ce sont là les seuls territoires de référence de populations se reconnaissant des attributs spécifiques, en dehors de la citoyenneté.

7 Mais en cette dimension micro de la territorialisation de groupe, en ces lieux, l'espace est à la fois partagé et peu signalisé. Les limites de la commune doivent être réaffirmées : subdivision étatique la plus fine, tout citoyen doit s'y reconnaître. Chaque groupe constitue ses propres référents et définit des spatialités spécifiques, parfois interstitielles. Tant physiquement que psychologiquement, tout nouveau marqueur, attribué de manière réelle ou virtuelle à un lieu, contribue à l'affecter, à le valoriser de manière singulière. Il est l'espace approprié des uns, au sens d'assemblage de lieux références de l'expression d'une identité singulière : en un Etat qui n'est pas (n'est plus) celui de la nation ${ }^{4}$. Quelles valeurs revêt-il pour les autres : pour celles et ceux qui en 
partagent les limites et les usages ? Une dynamique de groupe peut-elle faire sien cet espace du quotidien sans engendrer un «faire à part », un processus d'exclusion?

\section{Renouveaux identitaires : le lieu, affirmation d'une présence}

8 Pendant le communisme, chaque population (groupes de souches nationales ou confessionnelles spécifiques) avait ses quartiers de villes, ses bourgs ruraux. Hormis quelques cas, les identifiants majeurs d'une présence tenaient en une église ou un temple, en quelques institutions scolaires et, parfois, en des infrastructures (théâtres) ou association culturelles (Voiculescu, 1997). En 1989, des dissociations de ce type ne sont plus observables que dans des communes villageoises isolées.

9 Cette évolution repose en grande partie sur les choix politiques opérés pendant le communisme. Limitées bien que tolérées, les Églises perdirent leur fonction de pôle communal, ou du moins paroissial (Popa, 2000). Les communes, elles, ont été élargies à l'échelle d'un bourg pivot et de quelques bourgs annexes. Ceci a contribué à renforcer la transformation, sinon l'élimination, de multiples repères historiques (Von Hirshhausen, 1997). Caractéristiques jusque-là de la société roumaine, les regroupements villageois sur bases identitaires ne peuvent plus se maintenir que difficilement (Institut Interculturel de Timisoara, 1999-2001). Enfin, fournir à chacun un logement au plus près de son lieu de travail permet de rompre les regroupements sociaux, de les déterritorialiser.

10 Une historiographie s'est développée en parallèle de ces décisions gouvernementales, prônant la suprématie de la culture et des acquis roumains, "sublimant " la nation (Durandin, 1995). La part des perceptions encore liées à la propagande communiste est telle aujourd'hui que toute revendication identitaire autre que roumaine est difficilement acceptée, car contraire au «mythe de l'unité permanente de la Roumanie » (Boia, 2003). En somme, toute démarche de regroupement identitaire s'opposerait à l'idéal national roumain.

11 Le marquage n'est donc pas seulement propre au groupe social concerné. Il témoigne de la présence, de l'existence et de la pérennité de ce groupe aux yeux de tous. À diverses échelles, différentes stratégies peuvent être identifiées. Dans un contexte d'ouverture brutale, tel que celui de la société roumaine, le risque n'est-il pas grand, en retour, que cela engendre une appropriation, certaines formes de ségrégations? Affichages et discours, symboliques et usages, doivent à la fois entretenir et diffuser les valeurs d'un (ou plusieurs) groupe(s).

12 Ces difficultés sont particulièrement observables dans une région telle que le Banat. Là, face à la pluralité des groupes sociaux en place et à l'extrême interpénétration des populations, chaque objet et chaque acte prend valeur de symbole, comme marqueur d'une présence (Ancuta-Sirbovan, 1995). Pour autant, si une communauté ou un groupe, entend s'approprier son espace proche, le fait-il au détriment de la présence d'autres populations? 


\section{Nadlac, ville duale ? Une appropriation non exclusive?}

13 Nadlac est une petite ville/commune ${ }^{5}$ frontalière de la Hongrie, située à l'extrême nord $\mathrm{du}$ Banat roumain dont elle forme une des limites historiques (Bioteau, 2001) (fig. 1). La commune se compose de deux entités distantes de quelques centaines de mètres. Les infrastructures douanières, accompagnées des logements des douaniers, constituent un ensemble clos : son personnel étant recruté à l'échelle nationale, son peuplement est à majorité roumaine. Le bourg centre de la commune forme la seconde entité remarquable. Il est traversé par la route européenne opérant la jonction entre Budapest et Bucarest. Sa spécificité est d'abriter une population de souche slovaque majoritaire (tableau 1).

Dans le même temps, le développement du poste frontière et l'émergence de nouvelles perspectives économiques ont encouragé l'installation de résidents non slovaques. La division première entre espace douanier et bourg slovaque tend à se résorber. Des populations de souche roumaine (ou autres) prennent place en ville, tandis que la communauté slovaque locale peine à se renouveler suite aux départs des plus jeunes et sous l'effet de mariages mixtes.

Figure 1 : Éléments de localisation des villes d'étude Towns studied : Elements of Localisation

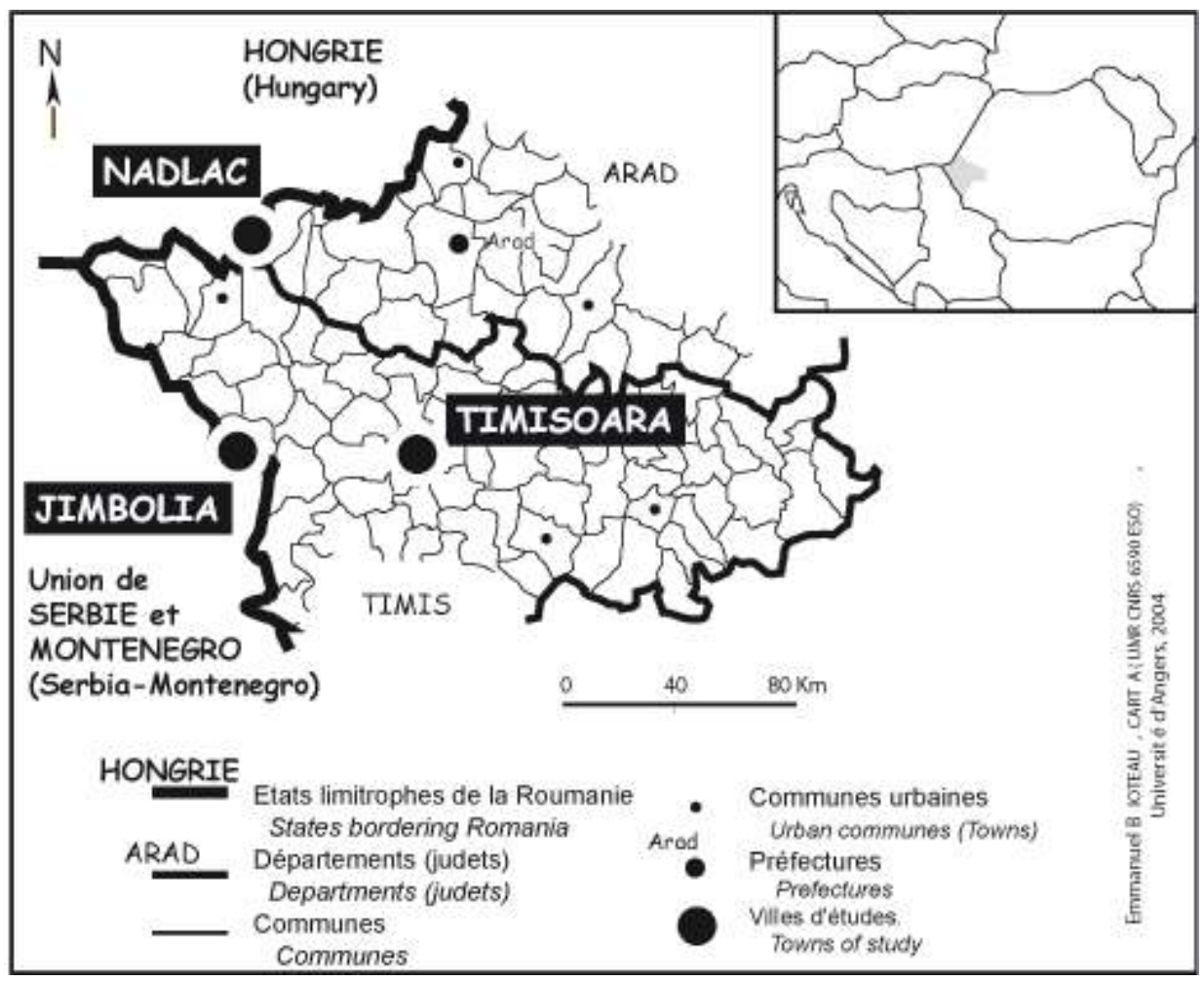


Tableau 1 : Groupes ethniques et confessions (exprimées) dans les trois villes d'étude, en 2002 Ethnic and Religious Groups (expressed by the individuals) in the Three Towns studied, 2002

\begin{tabular}{|c|c|c|c|}
\hline Villes/Towns & Timisoara & Jimbolia & Nadlac \\
\hline Population totale/Population & 317651 & 11115 & 8140 \\
\hline \multicolumn{4}{|c|}{ Principaux groupes ethniques/ethnic groups } \\
\hline Roumains/Romanians & 270528 & 8042 & 3620 \\
\hline Magyars/Hungarians & 25135 & 1640 & 290 \\
\hline Allemands/Germans & 7165 & 515 & non rens. \\
\hline Serbes/Serbs & 6271 & non rens. & non rens. \\
\hline Tsiganes (ou Roms) ${ }^{x} / R o m s$ & 3114 & 768 & 206 \\
\hline Bulgares/Bulgarians & 1224 & non rens. & non rens. \\
\hline Ukrainiens/Ukrainians & 813 & non rens. & non rens. \\
\hline Slovaques/Slovakians & 580 & non rens. & 3881 \\
\hline Juifs*/ Jews & 376 & non rens. & non rens. \\
\hline autres/others & 2454 & 150 & 143 \\
\hline \multicolumn{4}{|c|}{ Principales confessions/confessions } \\
\hline Orthodoxes/Orthodoxes & 255955 & 7155 & \\
\hline Catholiques/Catholics & 31832 & 2957 & \\
\hline Gréco-catholiques/Greek catholics & 4191 & 79 & \\
\hline Réformés/Reformed & 6194 & 188 & \\
\hline Luthériens/Lutherians & 337 & non rens. & \\
\hline Juifs*/Jews & 358 & non rens. & \\
\hline Baptistes $\%$ Baptists & 4780 & 64 & \\
\hline Adventistes $/$ Adventists & 684 & 55 & \\
\hline Pentecôtistes $/$ Penticostals & 8408 & 449 & \\
\hline Musulmans/Muslims & 949 & non rens. & \\
\hline autres/others & 5600 & 156 & \\
\hline
\end{tabular}

$x$ : un grand nombre de Tsiganes ne se déclarent pas comme tels lors des recensements, faussant les données (leur nombre réel est certainement de trois à cinq fois supérieur, selon les estimations les plus faibles ; il serait pour Timisoara de plus de 20000 individus, selon l'Institut Interculturel de Timisoara)

* : les Juifs sont considérés comme formant à la fois un groupe confessionnel et un groupe ethnique (d'après la nomenclature des recensements de la population roumaine) ; il est à noter qu'un certain nombre d'entre-eux, athées, mais d'origine juive s'insèrent dans cette catégorie « ethnique », ce qui donne des résultats variables, en fonction des champs observés.

: : ces trois Églises (Baptiste, Adventiste et Pentecôtiste) forment ce que l'on dénomme couramment Églises néo-protestantes.

Source : Recensement de la population et de l'habitat en Roumanie, 2002 (publié par l'INSSE, Bucarest)

$x$ : Every time there is a census of the population, the Tziganes do not declare themselves in real number, thus distorting the data (according to the lowest estimations their number is certainly thrice or five times hisher; there could be more than 20000 individuals in Timis, oara according to the Intercultural Institute of Timisoara. * : Jews are considered as forming a denominational group as well as an ethnic one (taking into account the nomenclature of censuses of Romanian population); it is important to note that a certain number of them, though atheistic of Jew origin, enter this "ethnic" category, a fact that gives various results commensurate with the observed fields. ${ }^{\circ}$ : These three churches (Baptist, Adventist and Pentecostal) are commonly called Neo-Protestant Churches. Source : Census of the population and settlement in Romania, 2002 (published by INSSE, Bucharest)

Par la langue, toujours parlée et enseignée, et par un référent confessionnel à l'Église évangélique (traditionnelle), les Slovaques maintiennent leur visibilité à l'échelon local; le culte évangélique est tenu en langue slovaque. Nadlac abrite notamment le siège du Parti des Slovaques et des Tchèques de Roumanie ${ }^{6}$ et la présidence de la Société Culturelle et Scientifique Ivan Krasko, défendant la culture slovaque à l'échelle étatique. Cette ville fut la première fondée par des colons slovaques (1803) 7 . Elle constitue, démographiquement parlant, le premier pôle urbain du peuplement slovaque en Roumanie. La ville est ainsi le site pivot de l'identitaire slovaque en Roumanie.

Pourtant, la ville se situe dans une région peu densément occupée par les Slovaques, par rapport à des espaces voisins (fig. 2). Aucune organisation des Slovaques n'a été rendue possible durant les quarante années de communisme. Les pressions exercées 
par les autorités communistes sur les institutions religieuses et les limites imposées à tout rassemblement de population ont, de fait, interdit toute action commune des Slovaques de Roumanie. Les références identitaires sont aujourd'hui fondées à l'échelle de chaque enclave de peuplement. C'est pourquoi, afin de réaffirmer sa primauté dans l'organisation des Slovaques de Roumanie, Nadlac se doit de recouvrer des attributs spécifiques.

Figure 2 : Le peuplement de souche nationale slovaque dans l'Ouest de la Roumanie Population of Slovak National Origin in Western Romania

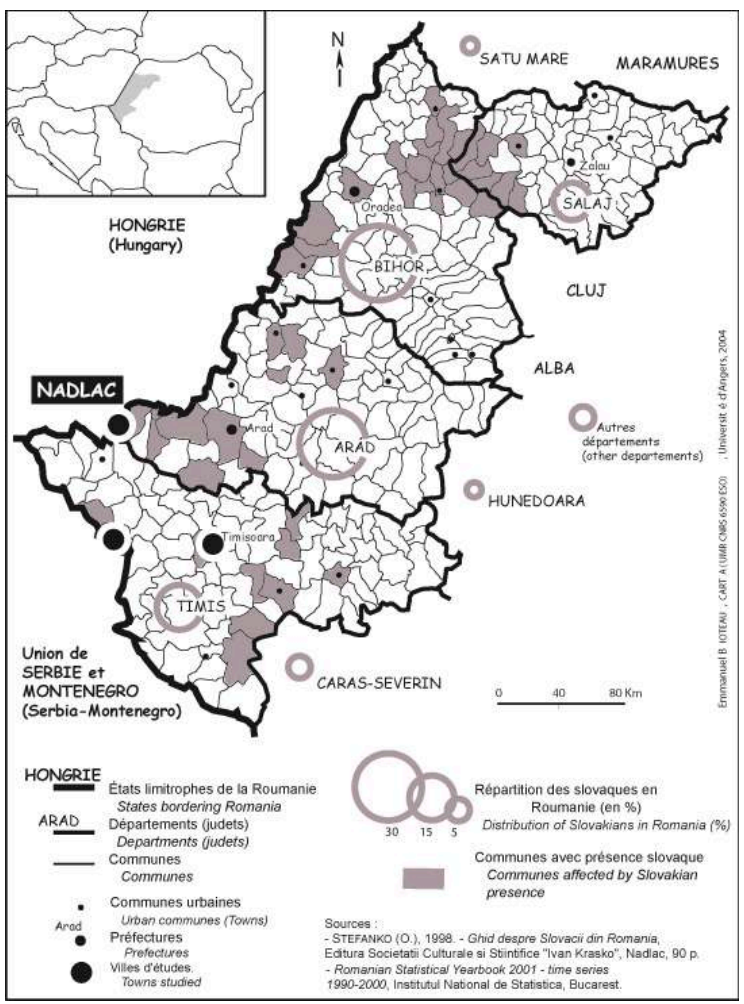

Dans ce contexte, la municipalité a organisé les 23 et 24 avril 2003 des cérémonies célébrant les 200 ans de présence slovaque à Nadlac (fig. 3). Les manifestations furent ouvertes à tous et ont engagé la paroisse évangélique aux côtés des autorités municipales et préfectorales. Des ensembles folkloriques et musicaux, des artisans, des écrivains, tous slovaques, de plusieurs origines géographiques, ont participé aux commémorations. Cependant, l'ensemble des habitants s'est mobilisé pour célébrer l'anniversaire de la fondation de la ville. Pour les uns, il s'agissait par cet événement de la commémoration de l'histoire et des personnalités locales. Tandis qu'à cet aspect se surimposait, pour les autres, une dimension identitaire, de valorisation du groupe ${ }^{8}$. 
Figure 3 : Extrait du journal Agenda du 19 avril 2003 (Timisoara) : « Événement à Nadlac pour la communauté slovaque "

Extract from the revue Agenda: "Event in Nadlac from the Slovakian Community"

\section{Eveniment la Nădlac}

- Pentru comunitatea slovacă

Comunitatea slovacă din Nădlac sărbătoreşte 200 de ani de la aşezarea în localitate, organizând o serie de manifestări aniversare. Sărbătoarea începe în $\mathbf{2 3}$ aprilie, cu o expoziție de broderii slovace şi picturi, urmată de spectacolul „Mărturia ființării noastre", sustinut de Ansamblul Sálašan. În 24 aprilie, ora 9,30 , coloana alegorică va porni de la Primărie spre biserica evanghelică, unde, la ora 11,00 , va incepe liturghia festivă, iar la ora 13,15 se va dezveli o placă $c-$ memorativă. De la ora 16,00 , se va desfăşura un program cultural la care iși vor aduce contributtia şi invitati din străinătate: corul slovac din Békéscsaba, un ansamblu din Voivodina, majoretele din Krompachi - Slovacia, alături de orchestra de mandoline din Peregul Mare și formația Senzus. De la ora 17 va avea loc vernisajul a două expoziții, iar la ora 21 va începe tradiționalul bal.

L. $\mathrm{S}$.

La communauté slovaque de Nadlac

célèbre 200 ans d'implantation dans la localité, organisant une série de manifestations anniversaires. La célébration débute le 23 avril par une exposition de broderies slovaques et de peintures, suivie du spectacle "Reconnaissance de nos existences», présenté par L'Ensemble SalasŠan. Le 24 avril, à $9 \mathrm{~h} 30$, une colonne allégorique partira de la mairie vers l'église évangélique, où, à $11 \mathrm{~h} 00$, débutera la liturgie festive, tandis qu'à $13 \mathrm{~h} 15$ sera inaugurée une plaque commémorative. À partir de $16 \mathrm{~h} 00$ débutera un programme culturel auquel des invités de l'étranger apporteront leur contribution: la chorale slovaque de Békéscsaba (Hongrie), un ensemble de Voïvodine (Serbie), les majorettes de Krompachi - Slovaquie, accompagnés de l'orchestre de mandoline de Peregul Mare (Roumanie) et de la formation Senzus. À partir de $17 \mathrm{~h} 00$ aura lieu le vernissage d'une seconde exposition, tandis qu'à $21 \mathrm{~h} 00$ débutera le traditionnel bal.

[version en langue française, Emmanuel Bioteau]

Ici, le groupe slovaque entend célébrer pour lui-même l'anniversaire de sa présence. Les festivités sont de courte durée, un site unique, et avec pour seuls invités (bien qu'étrangers) des groupes folkloriques slovaques. Dans le cas de Nadlac, la présence des Slovaques est ancienne; ils constituent encore le principal groupe de population de la commune. Les célébrations s'appuient tant sur la mairie (le maire est Slovaque) que sur l'église évangélique. Nous nous trouvons en présence d'une véritable communauté villageoise, soudée : les festivités ont pour objectif de réunir un maximum d'habitants, autour d'un référent historique commun. Ainsi se réaffirme l'identité de groupe et l'indication d'une primauté locale.

Trouver de telles informations, alors que slovaques ne sont pas potentiellement les principaux lecteurs, témoigne d'une ouverture culturelle, propre au Banat et à la région de Timisoara. Cela étant, force est de préciser que le directeur de publication (et le comité propriétaire) du journal est de souche magyare ; mais cette singularité n'est pas seule déterminante dans les choix de diffusion d'informations du quotidien. 
les Slovaques agissent pour eux-mêmes, ils utilisent l'image de Nadlac comme vecteur d'une identité spécifique. À ce titre, la mise en place d'une plaque commémorative le 24 avril 2003 ajoute un symbole de leur présence. Rappelant la fondation de la ville, cet objet s'adresse dans le même temps à l'ensemble des habitants de la commune'. Il en va de même pour de nombreux marquages internes dans Nadlac. Ainsi, les bâtiments se couvrent d'une forte densité d'écrits en langue slovaque: des dénominations de rues aux devantures commerciales. Toujours, les affichages se doublent de l'usage de la langue roumaine, afin que chacun, quel qu'il soit, puisse se repérer dans la ville ${ }^{10}$. des Slovaques et perçoivent les écrits en langue slovaque comme un attribut local. Leurs parcours en ville sont indépendants de ces marquages. Ils pratiquent indifféremment les commerces et les lieux, quelles qu'en soient les propriétaires ou les références identitaires associées. Leurs trajets sont induits par des conditions de proximité et liés à des relations amicales ou professionnelles.

Pour les Slovaques, la ville est aussi conçue comme un cadre ouvert. Pour leurs achats quotidiens, ils privilégient les commerces tenus par des Slovaques. Plusieurs de nos interlocuteurs confient pouvoir, par ce biais, maintenir l'usage de leur langue maternelle. Certains précisent qu'ainsi ils garantissent aux commerçants de pouvoir subsister localement; ce qui limite en partie l'émigration des plus jeunes. Mais ces commerces ne sont pas tous concentrés à proximité des lieux de mémoire des Slovaques dans la ville. Ainsi, l'espace urbain se subdivise en de nombreux pôles: église, bâtiments associatifs, sites commémoratifs (via la mise en place de plaques), commerces... Des parcours spécifiques prennent forme.

21 Ce mode de marquage de l'espace relève d'un modèle déjà décrit par de multiples observateurs, en d'autres occasions. Il n'est pas exclusif. Il participe bien d'une dynamique de valorisation non seulement communautaire mais plus largement communale. Chacun peut se retrouver dans les actions de promotion culturelle décidées par la municipalité (slovaque). En ce sens, s'il est une appropriation, celle-ci est propre aux Slovaques, entre eux. Il y a appropriation dans la mesure où les écrits (langue slovaque) ne sont pas accessibles aux autres... il y a partage (non exclusivité) du fait que les sites en question ne sont pas clos, et donc pratiqués par tous au quotidien. Cela interroge la notion d'appropriation. Peut-il y avoir appropriation sans exclusivité ?

\section{Innover pour perpétuer le groupe : être Magyar à Timisoara}

De la petite ville de Nadlac à la capitale régionale Timisoara, peu de recoupements semblent possibles. La première ne recense que deux grands groupes ethnolinguistiques pour une population n'excédant pas les douze mille habitants. La seconde, elle, est fréquemment décrite comme la ville multiculturelle par excellence de la Roumanie. Capitale du Banat, ou se côtoient une quinzaine de groupes ethnolinguistiques et autant de cultes, Timisoara en revêt les attributs sociaux (tableau 1).

23 Dans un contexte de grande ville, la dispersion des habitants limite une organisation en groupes sociaux ethnolinguistiques : la méconnaissance des individus entre eux freine 
tout processus. L'établissement de Magyars à Timisoara est ancien et démographiquement conséquent. Dans quelques quartiers centraux et péricentraux, une forte densité de foyers magyars a pu être maintenue. Cependant, aujourd'hui, de nombreux Magyars résident dans des ensembles de logements collectifs périphériques, où aucun attribut spécifique ne rappelle l'existence du groupe. Vecteurs de l'identité magyare, les Églises (catholicisme et protestantismes) ne disposent pas de lieux de culte dans ces quartiers construits en période communiste ; les densités de fidèles y restent faibles, rendant inutile tout nouveau bâti (Bioteau et Popa, 2004). Les écoles proposant des cursus en langue hongroise sont absentes de ces quartiers : elles restent proches des églises et des temples.

Toutefois, au regard du passé de la ville, les tenants d'une organisation de groupe ${ }^{11}$ entendent rappeler leur implication dans le processus de développement urbain: lorsque les Magyars se concentraient essentiellement dans trois quartiers du centre et des faubourgs de la fin du XIX siècle. Au cours des années quatre-vingt-dix, des rues commerçantes se sont réformées au sein des quartiers magyars ou considérés comme tels. Une partie des devantures exprime, par l'usage du hongrois, une identification des lieux. Au gré des disponibilités immobilières, ces commerces sont disposés à proximité des écoles et des églises de langue hongroise. Au centre, à proximité de la préfecture, une partie de l'ancien siège des représentations politiques et syndicales magyares de la ville est réinvestie par des associations magyares, un hebdomadaire en langue hongroise et l'organe politique local du groupe.

La présence magyare à Timisoara est illustrée, aux yeux des promoteurs du groupe, par deux places, que bordent pour chacune un lieu de culte en langue hongroise (fig. 4).

Figure 4 : Présences et marqueurs de magyarité à Timisoara, en 2003 Markers and Situations of Magyar National Origin Population in Timisoara, 2003

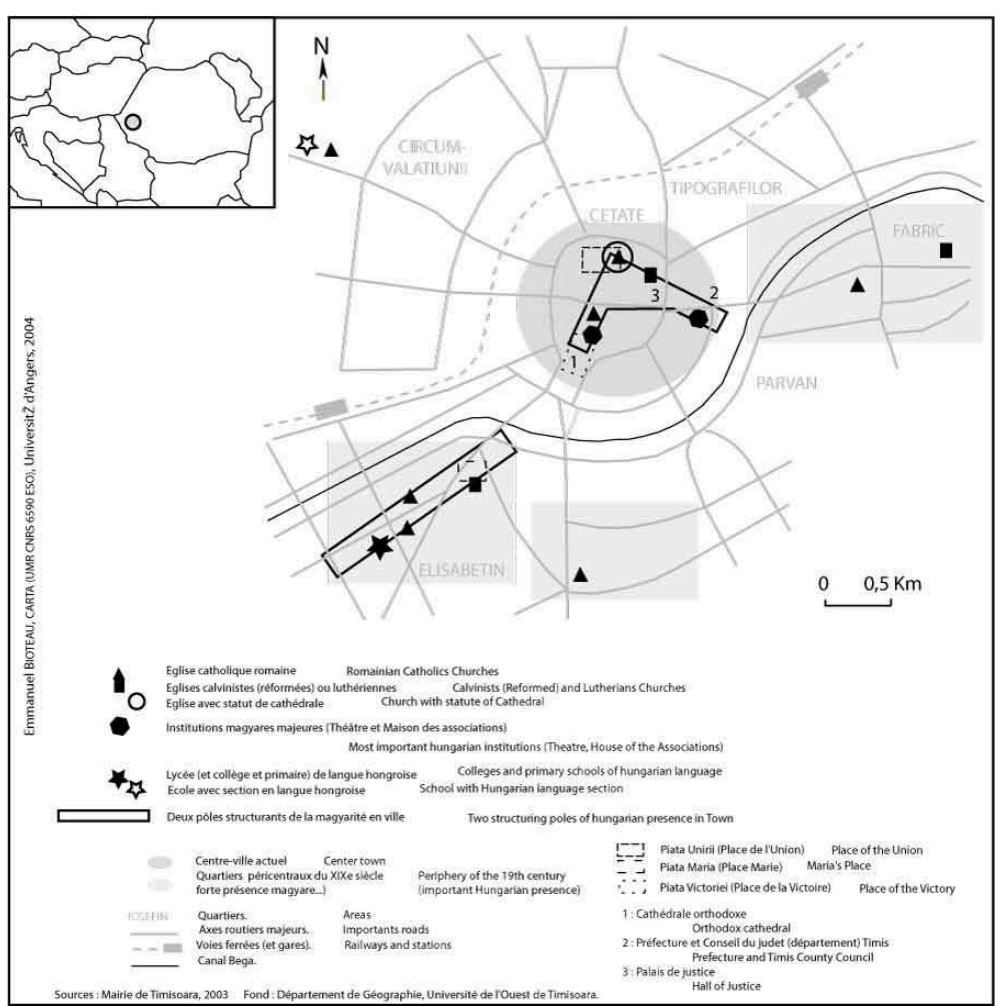


26 La Piata Unirii (Place de l'Union) est située au centre de la ville. La cathédrale catholique, affiliée à la présence allemande mais ouverte au culte en langue hongroise, y a été bâtie. S'ajoutent à la symbolique de ce lieu central les valeurs des institutions voisines, telle que le palais de justice de la ville, et le patrimoine architectural du quartier, datent de construction hongroise, de la seconde moitié du XIX siècle $^{12}$. La place constitue le site de refondation de la ville par les colons délégués par le royaume d'Autriche, à la fin du XVIII ${ }^{\mathrm{e}}$ siècle. Pour les Magyars de Timisoara, il s'agit d'un lieu témoignant d'une présence ancienne et d'un rôle dominant, aux côtés des populations allemandes ${ }^{13}$.

Plus encore, cette place constitue le vieux centre de la ville, celui de ses origines, en opposition à la place récente qui s'ouvre sur la cathédrale orthodoxe roumaine (Institut culturel de Timisoara, 2001). Cette dernière se referme sur le théâtre de la ville qui, se prolongeant en direction de la Piata Unirii, abrite dans l'une de ses ailes le Théâtre d'État Csiky Gergely, dont les pièces sont jouées en langue hongroise. Ainsi, un marquage fort naît en retrait de ce centre neuf, en direction de la cathédrale catholique. Le jubilée du théâtre en langue hongroise a suscité l'organisation de nombreuses festivités, réparties dans ces lieux phares de la magyarité $e^{14}$ (cathédrale, théâtre et bâtiment abritant les institutions magyares locales) (figure 5).

L'intérêt principal de l'autre place, la Piata Maria (place Marie), ne réside pas tant dans la présence du temple réformé, par ailleurs peu visible, que dans le souvenir de l'un de ses anciens officiants. L'Histoire retient que la Révolution roumaine a débuté à Timisoara suite à l'opposition de la population locale (Magyars et autres) à la mutation du pasteur Tökes Laszlo. La Piata Maria conserve de cet événement controversé la symbolique de lieu originel de la Révolution. En cet honneur, une plaque commémorative y a été apposée.

Pour les Magyars, cette place constituait déjà auparavant un pivot de leur présence en ville. Depuis lors, elle acquiert une symbolique supplémentaire, en s'imposant dans la société civile. À partir de cette place, en direction du lycée en langue hongroise de Timisoara, se développent de multiples commerces tenus par des Magyars. Cet ensemble urbain, composé de deux axes structurants, vient d'être rénové et préfigure l'une des extensions majeures du centre ville, au-delà de la cathédrale orthodoxe. Le passage du tramway dans cette rue n'est pas non plus étranger à la réactivation de son dynamisme, la ligne en question desservant la principale gare de la ville ${ }^{15}$. 
Figure 5 : Affiche pour le Jubilé du Théâtre Magyar ( $50^{\mathrm{e}}$ anniversaire) de Timisoara, du 3 au 9 mai 2003 (Double usage linguistique hongrois et roumain) (cl. E. Bioteau, 2003)

Notice from the Jubileum of the Hungarian Theatre of Timisoara (50th anniversary), may 3 to 9, 2003 (Double language markers Hungarian and Romanian)

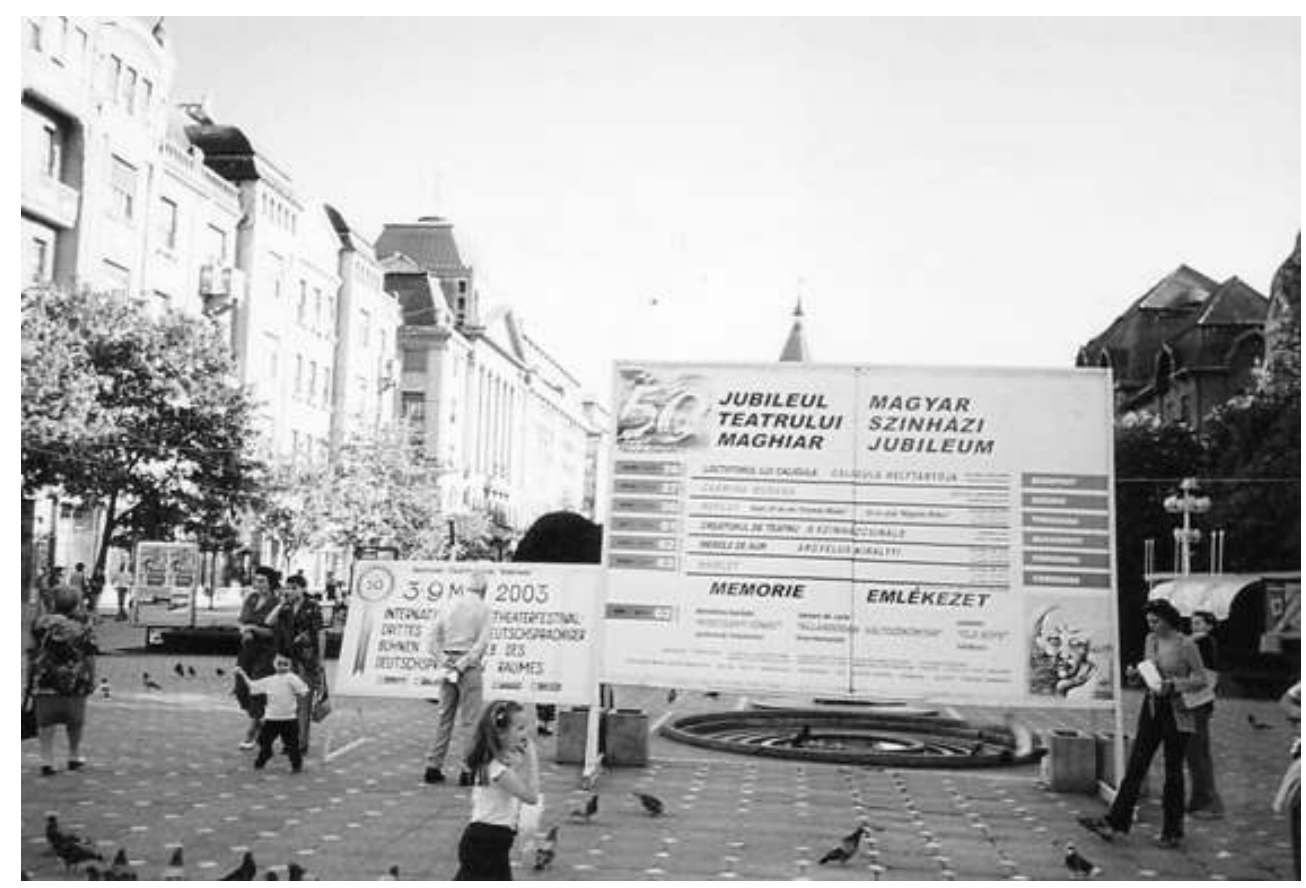

Cliché pris sur la place de la Victoire de Timisoara. En arrière-plan, la cathédrale orthodoxe. Au dos du photographe, l'opéra de la ville, lequel abrite le Théâtre Magyar d'État. Cette affiche rédigée dans les deux langues (roumain et hongrois) occupe une position centrale sur la place, et donc en ville. Une affiche similaire est placée à proximité de la cathédrale, pour qui porte son regard en direction de l'opéra. Ainsi, toute perspective est occupée par cette publicité sur la Place de la Victoire. Nous pouvons remarquer que quelques habitants passent aux côtés de l'affiche. Un vieil homme s'y intéresse. En bas de l'affiche principale est inscrit le terme « mémoire » (memorie, emlékezet). ॥ correspond à un spectacle/débat autour du thème de Trianon (Traité du même nom) et des différents conflits hungaro-roumains. L'événement culturel doit, aussi, permettre de mieux envisager l'altérité.

Cet exemple illustre la multiplication de marqueurs ponctuels en centre-ville et dans ses alentours, et non une opposition spatiale. Pour les Magyars désireux de maintenir leur attache au groupe, l'espace urbain se singularise en deux pôles, que les deux places évoquées contribuent à structurer. Ces éléments et ces marqueurs urbains (plaques commémoratives ou statues) imposent un parcours singulier ouvert à eux seuls, grâce à leur maittrise du hongrois et via leur confession. Pour autant, tout un chacun, Magyar ou autre, peut se déplacer librement au sein des espaces ainsi délimités : le marquage observé n'impose pas de cloisonnement. Toutefois se pose le problème du devenir exclusif de ce marquage, de cette dynamique de concentration des commerces, institutions, ou autres, en un espace assez précisément délimité. Le groupe agit pour lui-même et en adressant un message aux autres populations, le tout à l'échelle locale.

\section{Des symboliques sans appropriation ? Jimbolia}

31 Jimbolia est une petite ville située le long de la frontière de l'actuelle Union de Serbie et Monténégro (fig. 1). D'une population équivalente à celle de Nadlac, elle tranche avec cette dernière par son caractère pluriethnique. Là se côtoient une population de souche roumaine majoritaire, ainsi que des Magyars, des Rroms en assez grand nombre, 
quelques Serbes et des Allemands qui, durant l'entre-deux-guerres, constituaient la principale population de la ville, et ne forment plus aujourd'hui qu'un groupe âgé et fortement minoritaire (tableau 1).

Dans l'immédiat post-communisme, la municipalité de la ville fut confrontée à un manque cruel d'investissements étrangers, en partie lié à l'embargo imposé à l'exYougoslavie. L'un des reproches adressé à la ville par les investisseurs potentiels reposait, en dehors de la situation de la ville, sur l'état du bâti et la propreté urbaine. Depuis l'année électorale 2000 (élections municipales), une opération d'embellissement de la ville a été conduite. Elle consistait dans la plantation d'arbres et dans l'obligation pour chaque foyer de clôturer sa (ou ses) parcelle(s) urbaine(s). Afin de garantir la réussite de cette action, une taxe spéciale a été mise en place pour tout contrevenant.

Cette première initiative s'est accompagnée d'une réutilisation originale d'un stock vacant, en cours de décomposition dans une décharge sauvage, de vieux matériels électroménagers auparavant importés de Yougoslavie. En fonction des possibilités, les pièces ont été revendues à des ferrailleurs, ou recyclées en poubelles ou en éléments de mobilier urbain. La politique ainsi menée répondait tant aux besoins environnementaux (équipements urbains et nettoyage des décharges périphériques) qu'à l'inactivité constatée d'une partie croissante de la population ${ }^{16}$. Surtout, l'opération a eu pour effet de réguler certaines tensions interethniques. Les rapports sociaux entre tenants de différents groupes se dégradaient suite au départ des Allemands - ces derniers étant en somme les garants de l'identité locale ${ }^{17}$. Le cloisonnement ethnique de la population par quartiers s'accentuait.

Le maire ainsi que la majorité de son conseil ont été élus sur la liste de l'organe politique des Magyars de Roumanie (l'UDMR), bien que minoritaire. Cette élection reposait sur un programme électoral qui prônait un embellissement rapide et au moindre coût de la ville, susceptible en retour d'en accélérer le développement économique. La promesse tenue et la mobilisation de l'ensemble des habitants (de gré ou de force) permirent le rétablissement d'un dialogue interne constructif; alors qu'auparavant il menaçait d'être rompu.

Ces deux dernières années, des investissements étrangers ont été effectués dans la ville. Ces investissements soulignent aux yeux de la population l'apport de la politique mise en place et des efforts consentis ${ }^{18}$. Constat supplémentaire : lors des dernières élections municipales, de mai 2004, le maire et son conseil ont été réélus sans grandes difficultés. Dans une certaine mesure, les clivages identitaires et ethnolinguistiques ont pu être dépassés, au profit et en raison d'une dynamique économique recouvrée.

Cette politique municipale contribue à modifier certains usages au quotidien. Pour autant, nous pouvons nous interroger quant à la portée à long terme de telles actions. Elles permettent d'éviter l'émergence de comportements exclusifs. Mais elles ne modifient pas pour autant des usages de l'espace spécifiques aux groupes sociaux recensés, parce qu'elles n'affectent pas leur propre symbolique. Chacun des groupes en place lit différemment l'espace urbain. Sièges de partis politiques, musées (notamment un musée spécifique aux Allemands), lieux de cultes et autres institutions ou équipements jusqu'aux dénominations de rues, traduisent la présence des uns et des autres en ville. Les principaux groupes en place disposent de leurs propres marqueurs. Une question se pose ainsi de manière récurrente ${ }^{19}$, au sujet des enjeux réels de ces élections et des projets développés. S'ils adhèrent aux programmes de développement 
et d'embellissement de la ville, une partie des habitants de souche roumaine regrette la mainmise des Magyars (de quelques-uns d'entre eux, politisés) sur la vie politique et culturelle locale. Certains s'empressent de rappeler le rattachement de la ville à la Roumanie à la fin des années 1950, par échange territorial avec la Yougoslavie ${ }^{20}$. En somme, la symbolique identitaire de la ville se trouve renforcée par ces actions d'aménagements ponctuels.

Ce double rapport, d'abord au groupe et, ensuite, au lieu de vie, est souligné par la mise en place d'une statue du poète roumain Mihai Eminescu. Suite à son élection, le maire dont il est question a commandé l'édification d'un buste du poète, à proximité de l'hôtel de ville. Il se garantit ainsi du rejet, pour causes identitaires, d'une partie de la population locale ${ }^{21}$.

Un tel marquage d'une "roumanité » des lieux est identifiable dans de nombreuses petites villes et communes de l'Ouest roumain, où les statues (le plus souvent des bustes) de ce poète ou d'autres figures littéraires ou historiques roumaines ont été installées par les municipalités : soit durant la période communiste, soit comme c'est le cas dans le Banat, dans l'immédiat post-communisme. Cet acte contribue à symboliser l'appartenance du site au territoire de la nation roumaine, derrière la figure de l'un des plus grands promoteurs de l'identité roumaine (via ses écrits).

\section{Introduire usages et symboliques de l'espace dans une réflexion citoyenne}

41 Le marquage propre à un groupe (ethnoculturel) donné, à l'échelle des quartiers ou des petites villes, s'effectue a priori via d'autres vecteurs, telles les églises ou des référents propres au groupe d'appartenance. Ainsi, à Nadlac, chaque groupe en place jouit de certains attributs, sans que cela n'engendre de processus exclusif. Dans ce cas, les autorités locales agissent par la promotion d'une image d'ancienneté associée à une ouverture. De l'extérieur, la ville est perçue comme un espace marqué par une présence slovaque forte. L'activité culturelle y est telle que, ajoutée à une frontière ouverte, Nadlac dégage une impression de prospérité ${ }^{22}$. Par ce biais, elle s'impose aux Slovaques de Roumanie. Pourtant, elle est perçue comme une ville en déclin par les populations des communes avoisinantes.

Ces marques présentes dans la ville lui confèrent une identité spécifique. Elles sont susceptibles de servir tant la communauté slovaque locale, qui ainsi se pérennise, que l'ensemble des habitants de la commune. Le marquage de l'espace urbain est défini suivant deux niveaux de lecture: des parcours des populations d'autres souches nationales au parcours identitaire des Slovaques. Dirigée par un maire issu du Parti des Slovaques et Tchèques de Roumanie, la municipalité entend défendre l'image et les spécificités locales. L'acceptation de ces objectifs par l'ensemble des habitants de la ville paraît primordiale dans la justification de telles formes de marquages, oscillant entre appropriation (dans le groupe) et non exclusivité (auprès de tous citoyens).

De nombreux défis attendent la Roumanie, notamment son intégration future dans l'Union Européenne. C'est pourquoi dans ce contexte il sera nécessaire que le dialogue social et interculturel se renforce. L'une des hypothèses, à laquelle cette réflexion aboutit, repose sur la capacité des acteurs identifiés à maintenir ce dialogue, face à des intérêts concrets, économiques et individuels. 

groupes concernés. Attachements aux lieux, identifications, ne se révèlent pas exclusifs de façon systématique. Il en naît une ambivalence. L'espace est partagé, et en ce sens non approprié, car praticable par tous au quotidien. Ainsi, les groupes ethnoculturels (ou confessionnels) ne créent pas de ruptures physiques. Pourtant, ils marquent 
l'espace, produisent des territorialités identifiées par eux seuls. L'appropriation est alors symbolique. Elle permet d'afficher dans l'espace une reconstruction identitaire, non pas tant liée aux groupes sociaux en actions (politique, associative) qu'aux projets et intérêts individuels.

L'étude montre un dépassement du simple marquage, au profit d'une lecture différenciée de lieux donnés. Slovaques, Magyars et autres montrent des processus de spatialisation identitaires. Au contraire de nombre d'idées reçues, ils ne s'enferment pas dans leurs quartiers, dans leurs rues - du moins dans l'espace banatéen ici abordé. Leur mode d'occupation de l'espace recouvrent à la fois les notions de pratiques/usages et d'appropriation. Un entre-soi ouvert aux Autres? Une altérité permettant de construire en commun, plutôt que de produire de l'enfermement.

\section{BIBLIOGRAPHIE}

ANCUTA-SIRBOVAN (C.), 1995. - « Le Banat, archétype de la région multiethnique en Europe », dans GOETSCHY (A.), SANGUIN (A.-L.) (dir.), Langues régionales et relations transfrontalières en Europe, Paris, L'Harmattan, coll. « Géographie et Culture », p. 121-130.

Anuarul statistic al României 2001 - serii de timp 1990-2000 (Romanian Statistical Yearbook 2001 - time series 1990-2000). - Institutul National de Statistica, Bucarest, (document informatique).

BIOTEAU (E.), 2001. - Familles et institutions magyares dans le Banat roumain. Essai de définition d'une "magyarité », Mémoire de DEA Régulations sociales (géographie), Université d'Angers, 89 p. BIOTEAU (E.), POPA (N.), 2004. - « Pluriconfessionnalité et recompositions urbaines, le difficile équilibre. Réflexions sur l'ouest roumain : Timisoara, Arad et Satu Mare ", Annales de la Recherche Urbaine, «Urbanité et liens religieux », $\mathrm{n}^{\circ}$ 96, p. 62-72.

BoIA (L.), 2003. - La Roumanie. Un pays à la frontière de l'Europe, Paris, Les Belles Lettres, 415 p. Centre culturel françAis de timisoara, fundatia A treia EuRopa, 2001. - Scènes de vie. Mémoire et diversité culturelle. Timisoara, 1900-1945, Timisoara, 130 p.

DURANDIN (C.), 1995. - Histoire des Roumains, Paris, Fayard, 573 p.

HIRSCHHAUSEN (B. von), 1997. - « Le territoire roumain dans la turbulence. Transition politique et mutations géographiques », dans DUMORTIER (B.) (coord.), L'Europe médiane en mutation, Paris, Éditions du Temps, coll. «Questions de Géographie », p. 127-143.

INSTITUT INTERCULTUREL DE TIMISOARA, 1999-2001. - Minorités : identité et coexistence, IIT, Programme des Mesures de Confiance du Conseil de l'Europe, Timisoara, extrait du site [www.intercultural.ro]. POPA (N.), 2000. - «Identitate, teritorialitate si prestigiu cultural in spatiul geografic romanesc [Identité, territorialité et prestige culturel dans l'espace géographique roumain] », dans Regionalism and Integration. Culture, Space, Development. Regional Conference of Geography - Timisoara, Timisoara/Tübingen/Angers, p. 87-96.

SCHNAPPER (D.), 1998. - La relation à l'autre. Au cœur de la pensée sociologique, Paris, Gallimard, coll. « NRF Essais », $562 \mathrm{p}$. 
VERNICOS-PAPAGEORGIU (S.), REY (V.), 1994. - « Religions, nationalités et discontinuités territoriales. Le cas roumain en 1992 », L'Espace géographique, tome XXIII, n² 4, p. 300-311.

VOICULESCU (s.), 1997. - « The cultural landscape of the cities during transition - the case study of Western Transylvania, (titre en anglais, article rédigé en langue roumaine) », Analele Universitatii de Vest din Timisoara, seria Geografie, Vol. VII, p. 93-99.

\section{NOTES}

1. Lequel a donné lieu à nombre d'études économiques et politiques formant aujourd'hui une bibliographie vaste et complète, que nous n'éludons pas, mais qui relève d'un autre propos.

2. Le droit d'association est similaire à celui spécifié par le droit français (Loi 1901). Il est régi par la Constitution: Loi de libre association, Constitution de la République de Roumanie, 1991, Chapitre II, article 37.

3. Notamment Allemands et Juifs. Ces derniers sont considérés comme "groupes ethniques » d'après la nomenclature du Recensement de la Population de la Roumanie.

4. Le concept de nation ne sera pas débattu ici. Nous en faisons usage au sens de regroupement ethnoculturel et linguistique, admettant le flou d'une telle acception.

5. Le découpage territorial de la Roumanie regroupe en communes des bourgs centres et leurs satellites ruraux. Il différencie aussi les communes proprement dites, de caractères ruraux/ agricoles, et les villes, communes dont le bourg centre dispose d'attributs urbains (commerces, banques, infrastructures sanitaires, scolaires, militaires, etc.). Dans la nomenclature roumaine, Nadlac forme une ville, composée d'un seul bourg; fait rare en Roumanie où la plupart des communes (ou villes) associent plusieurs bourgs.

6. Organe politique réunissant deux groupes de souches nationales proches suivant le modèle offert par l'ancienne République de Tchécoslovaquie. La scission de cette dernière n'a pas eu de conséquence majeure sur cette représentation des deux groupes en Roumanie. Création en 1990.

7. Quelques localités ont été fondées antérieurement, sans toutefois ne jamais disposer du statut de ville en Roumanie, telle Mocrea (département Arad) en 1747.

8. Série d'entretiens réalisés à Nadlac, et dans le nord-est de la Roumanie auprès de personnes de souche slovaque, en avril 2003 et février 2004.

9. Il stipule la date de fondation de la paroisse évangélique, l'origine des habitants de Nadlac (provenant de la région de Bekescsaba, dans l'actuelle Hongrie) et affirme que Nadlac est une ville libre et ouverte à tous depuis 200 ans.

10. De plus, l'identité des commerçants n'est pas un handicap, dans la mesure où chacun est apte à s'exprimer dans les deux langues; ils sont toutefois pour la plupart de souche slovaque : ils possèdent le patrimoine bâti originel, leur permettant de développer leur activité à moindres frais. Le double affichage ici décrit est imposé juridiquement dans le domaine étatique et dans l'administration publique. Il est conseillé et non imposé pour les personnes et les sociétés de capitalisation privée.

11. Partis politiques (réunis au sein de l'UDMR, auquel s'ajoute depuis peu un organe autonomiste essentiellement actif dans le centre et le nord-ouest de la Roumanie), quelques associations et des personnalités locales (intellectuels, scientifiques, politiques, etc.).

12. Reconstruction partielle du centre ville, et extension, permettant de créer de nouveaux bâtiments administratifs (dont le palais de justice), des villas de riches commerçants magyars, etc. Seules quelques rues et places d'architecture allemande ont été conservées: preuve supplémentaire d'un marquage magyar dans la ville.

13. Les Allemands représentent le modèle d'une population parvenue à unifier et à mettre en valeur la région, tout en lui léguant un patrimoine architectural et industriel de premier ordre. 
Tous voient dans le départ de la plupart des Allemands une perte majeure pour la ville et pour sa région.

14. Magyarité, que nous définissons comme « sentiment d'appartenance » associé à des attributs identitaires spécifiques aux populations de langue hongroise et à l'histoire du peuple Magyar, ainsi qu'au cadre territorial de l'ancienne Grande Hongrie, d'Étienne le Grand à la double monarchie austro-hongroise au sein de l'Empire des Habsbourg.

15. Cette ligne de tramway fut aussi la première entièrement rénovée par la municipalité de Timisoara, via sa Régie de transports. Le programme consistait à rénover l'ensemble du réseau, et s'est achevé fin 2004.

16. Les plus défavorisés reçoivent un salaire, toutefois relativement faible, en échange de quelques heures hebdomadaires consacrées à l'entretien de la ville et à la bonne tenue des plantations.

17. Ce sont des Allemands qui ont fondé la ville et en ont assuré le développement économique. Les familles allemandes, alors majoritaires, ont assumé le pouvoir municipal jusqu'à l'avènement $\mathrm{du}$ communisme. L'ensemble des groupes ethnolinguistiques en place s'accordent pour reconnaître que leur départ affecte les liens inter-groupes : il n'y a plus d'ordre établi.

18. Bien que d'autres raisons motivent en réalités ces venues d'entreprises de capitalisations étrangères, telles l'ouverture croissante de l'économie roumaine, sa stabilité, et la situation, avantageuse à long terme, de Jimbolia.

19. Constat effectué au cours des divers entretiens menés à Jimbolia, entre 2001 et 2004.

20. Quelques bourgs ruraux à majorité serbe de Roumanie ont été cédés contre la ville de Jimbolia, au peuplement alors mixte roumain et allemand. Il s'agit de la dernière modification de frontière de la Roumanie.

21. Entretien du 27 juin 2002, en mairie de Jimbolia.

22. Effets de perceptions car la ville n'a pas su capter de retombées des transits, contrairement à des communes voisines; mais Nadlac est toujours le principal pôle économique de peuplement slovaque de Roumanie.

23. Cet affaiblissement de la plupart des groupes ethnolinguistiques doit être opposé à la forte croissance démographique des Rroms.

24. Si tant est que l'on puisse définir une magyarité, il est possible d'opérer une détermination similaire pour le peuplement de souche slovaque.

25. Entretien du 16 février 2004, quelques mois avant les élections législatives et municipales de Roumanie.

\section{RÉSUMÉS}

Durant une quarantaine d'années, les autorités communistes roumaines se sont attachées à limiter les regroupements sociaux. Les liens linguistiques, confessionnels ou de souches nationales partagées étaient ignorés. Seule comptait la citoyenneté. La réactivation de ces rassemblements constitue l'une des principales expressions sociales issue du changement de régime de 1989. Cependant, peu des témoins de la période antérieure au communisme subsistent. Ce processus de recomposition de groupements sociaux doit ainsi s'accomplir dans un cadre (de libre expression) mal connu.

Dans le Sud-Ouest de la Roumanie, chaque acteur, groupe ou institution, marque l'espace selon 
ses propres critères et objectifs. Il s'en suit des superpositions de parcours et la multiplication de signifiants. Dans le cas où ces pratiques s'accompagnent d'une prise en compte de l'autre, elles complètent les processus de recomposition sociale et spatiale. Les appropriations peuvent se révéler non exclusives. Sont-elles en ce sens de simples marquages? L'article ouvre un débat.

Over the last forty years, Communist Romanian authorities have been devoting their time to limiting social groupings of companies. Linguistic, religious or national background links that had been shared had to step aside in favour of citizenship criteria. The reactivation of these groupings constitutes one of the main social expressions born from the regime change in 1989. However few witnesses of the Communist period have lived on. This process of re-establishment of social groupings has, thus, to be carried out in an unknown framework (of free expression).

In the South-West of Romania, each actor, group or institution marks the space according to its own criteria and aims. Additional superpositions of journeys and multiplications of significants result from this. When these practices are accompanied by an awareness of the other, of a "constructive otherness", they complete the processes of social and spatial re-composition. Appropriations may appear as non-exclusive. Are they only space markers? This article tries to open a debate on these facts.

INDEX

Mots-clés : appropriation de l'espace, local, marquage, société civile, souche nationale Keywords : appropriation of space, civil society, local, national backgrounds, space markers Index géographique : Roumanie

\section{AUTEUR}

EMMANUEL BIOTEAU

CARTA (Centre angevin de recherche sur les territoires et l'aménagement) - Université d'Angers, ESO, UMR 6590 - CNRS, emmanuel.bioteau@univ-angers.fr 